\title{
Spatiotemporal statistical analysis of influenza mortality risk in the State of California during the period 1997-2001
}

\author{
Kyung-Mee Choi $\cdot$ Hwa-Lung Yu $\cdot$ Mark L. Wilson
}

Published online: 7 July 2007

(C) Springer-Verlag 2007

\begin{abstract}
Using the Bayesian maximum entropy (BME) method of spatiotemporal statistics, the present study examines the geographical risk pattern of influenza mortality in the state of California during the time period 1997-2001. BME risk analysis is of considerable value, since influenza is the largest contributing factor to wintertime mortality increases in the US. By incorporating age-adjusted mortality data collected at the county level, informative influenza mortality maps were generated and composite space-time influenza dependences were assessed quantitatively. On the basis this analysis, essential risk patterns and correlations were detected across the state during wintertime. It was found that significantly high risks initially occurred during December in the west-central part of the state; in the following two weeks the risk distribution extended in the south and east-central parts of the state; in late February significant influenza mortalities were detected mainly in the west-central part of the state. These findings, combined with the results of earlier works, can lead to useful conclusions regarding influenza risk assess-
\end{abstract}

\section{K.-M. Choi}

Department of Environmental and Occupational Health, University of North Texas Health Science Center,

3500 Camp Bowie Blvd., Fort Worth, TX 76107-2699, USA

\section{H.-L. Yu ( $₫)$}

Department of Bioenvironmental Systems Engineering,

National Taiwan University, 1, Sec 4, Roosevelt Rd,

Taipei 10617, Taiwan

e-mail: hlyu@mail.sdsu.edu

M. L. Wilson

Department of Epidemiology, School of Public Health, University of Michigan, 109 Observatory St.,

Ann Arbor, MI 48109-2029, USA ment in a space-time context and, also, point toward promising future research directions.

Keywords Influenza - Mapping - Mortality - Risk · Spatiotemporal statistics $\cdot$ BME $\cdot$ California

\section{Introduction}

Influenza epidemics typically occur during wintertime and have been responsible for an average of 36,000 deaths per year in the US during the period 1990-1999 (Harper et al. 2004). On a global scale, the World Health Organization (WHO) has suggested that there is currently a diminishing window of opportunity to stop the first massive outbreak of influenza in the twenty-first century. Influenza $A$ H1N1, $A$ $\mathrm{H} 3 \mathrm{~N} 2$ and $B$ viruses have been in global circulation since 1977. Most studies suggest that H3 changes more rapidly than $\mathrm{H} 1$, while influenza $B$ evolves more slowly than either $A$ subtype (Ferguson et al. 2003). Influenza epidemics continue to occur regularly with serious effects on the population health and the national economies, and an accurate quantitative assessment of these influenza effects is an important issue.

Evaluating influenza effects in terms of the population mortality distribution across space and time is an important matter (Lui and Kendal 1987). There has been evidence in the past that geographical space is, indeed, relevant to the pattern of influenza spread, and that the disease propagates in the form of spatial waves originated at epidemic centers (Cliff et al. 1986; Cliff and Haggett 1988; Cliff 1995; Haggett 2000). There is a disagreement, however, on whether the local dynamics of an epidemic characterized by its infection force or basic reproduction number depend on population density heterogeneity (May and Anderson 
1984; Anderson and May 1991). Such dependence may turn out to be quite complex. Non-homogeneous population density distributions have been considered in spatial clustering studies (Besag and Newell 1991; Openshaw and Charlton 1987; Openshaw et al. 1988; Cuzick and Edwards 1990). However, what is lacking is a systematic epidemic forecasting methodology that could be used by public health authorities to design an adequate operation plan of health care facilities during winter influenza outbreaks (Viboud et al. 2003).

In practice, it is not easy to find good spatial or spatiotemporal datasets at a reasonable mapping scale (e.g., Earn et al. 2002). Understanding the influenza dynamics solely in a temporal context makes it impossible to determine the composite space-time spread of the disease. In realistic spatial and/or temporal studies, one often needs to integrate multiple sources of information under conditions of uncertainty (e.g., Christakos et al. 2002; Golledge 2002). The Bayesian maximum entropy (BME) method of spatiotemporal modelling and mapping (Christakos 1998, 2000; Christakos et al. 2005) is an intelligent geographical information science construction that satisfies epistemic cognition ideals and logical inference rules (information maximization, knowledge synthesis, stochastic deduction and induction, etc.). The present study uses this powerful method to study the composite space-time risk patterns of influenza mortality in the state of California. The findings of the study, combined with those of earlier works (e.g., Choi et al. 2006), can provide useful information for efficient risk management and health decision-making.

\section{Materials and methods}

\subsection{Data sources: disease and age groups}

California mortality data were obtained from the Center for Health Statistics (DSMF 1997-2001). Records of deaths attributed to viral pneumonia and influenza were collected and categorized using the International Classification of Disease nineth (ICD-9) and tenth (ICD-10) codes for mortality data obtained during the years 1997-2001. We selected death rate information at the county level. We also used US census data for the years 1990-2000 to generate

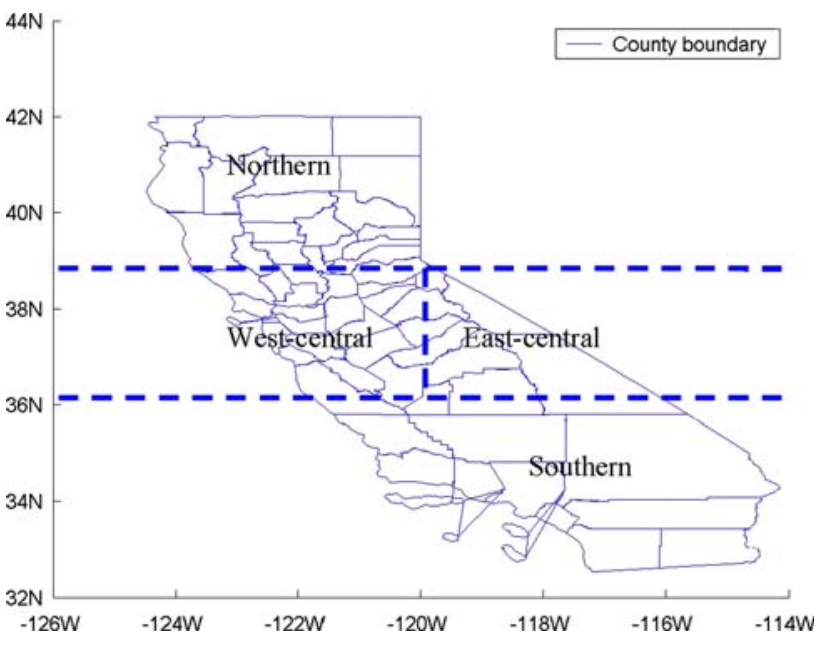

Fig. 1 A California county map

county-based mortality rates adjusted for the age group $a g \geq 65$ consisting of individuals of at least 65 year old. It is generally assumed that influenza viruses attack all age groups, but incidents of serious illness or even death are highest among older age groups and groups with preexisting medical conditions. These population groups are at increased risk to suffer complications due to influenza, such as bacterial infection. Herein, when we refer to influenza mortality we will mean mortality due to both viral pneumonia and influenza.

Our focus is the study of spatiotemporal patterns of mortality risk due to the influenza virus during wintertime (December through February). For this purpose, we assumed a study period consisting of 15 temporal points. Because influenza infection itself does not lead to immediate death, to each temporal point we assigned a bi-week unit period (herein denoted as $2 w i$ ). Since each year is divided into $2 w i$ units, the winter season considered in this study is determined by the time period September 10 of current year through April 8 of next year (see Table 1). Figure 1 presents a map of the 58 counties in the state of California. As is shown in this figure, the study area is divided into the north, central-west, central-east, and south regions in terms of the latitudes $36^{\circ} \mathrm{N}$ and $39^{\circ} \mathrm{N}$ and the longitude $-120^{\circ} \mathrm{W}$.

Table 1 The bi-weekly (2wi) time periods

\begin{tabular}{|c|c|c|c|c|c|c|c|c|c|c|c|c|c|c|c|}
\hline Time $(t)$ & 1 & 2 & 3 & 4 & 5 & 6 & 7 & 8 & 9 & 10 & 11 & 12 & 13 & 14 & 15 \\
\hline $\begin{array}{c}\text { Starting } \\
\text { date }\end{array}$ & Sep. 10 & Sep. 24 & Oct. 8 & Oct. 22 & Nov. 5 & Nov. 19 & Dec. 3 & Dec. 17 & Jan. 1 & Jan. 15 & Jan. 29 & Feb. 12 & Feb. 26 & Mar. 12 & Mar. 26 \\
\hline $\begin{array}{c}\text { Ending } \\
\text { date }\end{array}$ & Sep. 23 & Oct. 7 & Oct. 21 & Nov. 4 & Nov. 18 & Dec. 2 & Dec. 16 & Dec. 31 & Jan. 14 & Jan. 28 & Feb. 11 & Feb. 25 & Mar. 11 & Mar. 25 & Apr. 8 \\
\hline
\end{tabular}




\subsection{Spatiotemporal BME mapping}

Modern spatiotemporal statistics was introduced by Christakos (1990, 1991, 1992). Subsequent works include Goodall and Mardia (1994), Haas (1995), Bogaert (1996), Christakos and Bogaert (1996), Christakos and Hristopulos (1998), Kyriakidis and Journel (1999), and Cressie and Huang (1999); see relevant literature. Methodologically, influenza mortality rates are represented as Spatiotemporal Random Fields (S/TRF) and the BME method is subsequently implemented to map these rates across space and time. The BME method consists of three stages with a different Knowledge Base (KB) considered at each stage: the general $\mathrm{KB}, \mathcal{G}$ (core knowledge about the disease, including theoretical models and scientific laws), the specificatory $\mathrm{KB}, \mathcal{S}$ (case-specific hard and soft, instrument- and/or survey-based data), and the integration $\mathrm{KB}, \mathcal{K}$ (which is the union of the preceding general and specificatory $\mathrm{KB}$ ). BME accounts rigorously for the uncertainty features of the epidemic system in a composite spacetime domain; imposes no restriction on the shape of the probability distributions and the form of the predictor (non-Gaussian distributions and non-linear predictors are automatically incorporated); assimilates databases that are not necessarily disease variables, thus involves additional information sources; allows the consideration of multiplepoint stochastic moments of the epidemic variables across space-time; and derives several previous techniques of epidemic modelling (e.g., statistical regression) as its special cases. In light of these important features, the BME mapping of the mortality risk distribution is more informative and realistic than the maps generated by previous methods that do not possess the theoretical and applied qualities of BME (most of these methods can use only hard datasets; linearity and normality restrictions apply).

Let us represent the influenza mortality rate as the S/TRF $X(\boldsymbol{p})$ defined at each space-time point $\boldsymbol{p}=(s, t)$, where the vector $s$ denotes spatial location and $t$ is time. Our influenza modelling approach follows the BME stages:

a. At the structural stage, a probability density function (PDF), $f_{\mathcal{G}}\left(\chi_{\text {map }}\right)$, is constructed on the basis of the $\mathcal{G}-\mathrm{KB}$; the vector $\chi_{\text {map }}$ denotes a possible realization of the S/TRF associated with the point vector $\boldsymbol{p}_{\text {map }}$. The $\boldsymbol{\chi}_{\text {map }}$ generally includes hard data (exact measurements) $\boldsymbol{\chi}_{\text {hard }}=\left(\chi_{1}, \ldots, \chi_{m_{h}}\right)$ at points $\boldsymbol{p}_{\text {hard }}=\left(\boldsymbol{p}_{1}, \ldots, \boldsymbol{p}_{m_{h}}\right)$, soft data (uncertain observations) $\chi_{\text {soft }}=\left(\chi_{m_{h}+1}, \ldots\right.$, $\left.\chi_{m}\right)$ at points $\boldsymbol{p}_{\text {soft }}=\left(\boldsymbol{p}_{m_{h}+1}, \ldots, \boldsymbol{p}_{m}\right)$, and the unknown estimates $\chi_{k}$ at points $\boldsymbol{p}_{\boldsymbol{k}}$.

b. At the specificatory stage, the $\mathcal{S}-\mathrm{KB}$ considers hard data and/or soft data (in the form of intervals and probability distributions). Table 2 displays some examples of soft data; the $F_{\mathcal{S}}$ denotes the cumulative distribution function (CDF) derived from the $\mathcal{S}-\mathrm{KB}$ at the soft data points, $\boldsymbol{I}$ is a vector of mortality interval values at the same (soft data) points, and $\boldsymbol{I}_{k}$ is a vector of interval values at the estimation points.

c. At the integration stage, the $\mathcal{G}$ - and $\mathcal{S}-\mathrm{KB}$ are combined to yield the integration $\operatorname{PDF} f_{\mathcal{K}}\left(\chi_{k}\right)$ at each mapping point $\boldsymbol{p}_{\boldsymbol{k}}$ using the operational Bayesian formula

$f_{\mathcal{K}}\left(\chi_{\boldsymbol{k}}\right)=A^{-1} \int_{\boldsymbol{D}} d \Xi_{\mathcal{S}}\left(\boldsymbol{\chi}_{\mathrm{soft}}\right) f_{\mathcal{G}}\left(\chi_{\text {map }}\right)$

where $A$ is a normalization constant, and the $\Xi_{\mathcal{S}}$ and $\boldsymbol{D}$ denote, respectively, an integration operator and the range determined by the $\mathcal{S}-\mathrm{KB}$. Table 2 shows some examples of $\Xi_{\mathcal{S}}$ and $\boldsymbol{D}$ associated with the displayed soft data. From the integration PDF (1), mortality estimates for $\chi_{k}$ can be readily derived across space and time. E.g., the mode estimate is obtained by maximizing $f_{\mathcal{K}}\left(\chi_{k}\right)$. The mean estimation error can be also calculated from $f_{\mathcal{K}}\left(\chi_{k}\right)$ at each space-time mapping point.

\subsection{Spatiotemporal risk assessment}

To detect spatiotemporal risk patterns in the spread of influenza, we defined influenza mortality risk as a function of the size of the local area in which influenza mortality estimates are above a certain threshold (expressed in number of deaths). The geographical domain $(R)$ of interest, i.e. the state of California, is partitioned into the regions $\left(R_{i}\right)$ so that the $R_{i}$ do not overlap with one another (i.e., $R_{i} \cap R_{j}=\emptyset$ for $i \neq j$ ). Then, the normalized influenza mortality risk in region $R_{i}$ and time $t$ is defined as (in \%)

$\operatorname{Risk}\left(R_{i}, t\right)=\frac{A\left(R_{i}, t\right)}{A(R, t)} \times 100$,

where $A\left(R_{i}, t\right)$ is the area size in region $R_{i}$ in which influenza mortality exceeds a threshold at time $t$. Clearly, the $\operatorname{Risk}\left(R_{i}, t\right)$ field may vary with time and across geographical space.

Using the definition (2) of influenza mortality risk, space-time maps of $\operatorname{Risk}\left(R_{i}, t\right)$ can be produced, and the local $R_{i}$-contributions to the global (e.g., statewide) influ-

Table 2 Examples of soft data with the associated $\boldsymbol{D}$ and $\Xi_{\mathcal{S}}$

\begin{tabular}{lll}
\hline Soft data & $D$ & $\Xi_{\mathcal{S}}\left(\chi_{\text {soft }}\right)$ \\
\hline Interval & $I$ & $\chi_{\text {soft }}$ \\
Probabilistic & $I$ & $F_{\mathcal{S}}\left(\chi_{\text {soft }}\right)$ \\
Functional & $I \cup I_{k}$ & $F_{\mathcal{S}}\left(\chi_{\text {soft }}, \chi_{k}\right)$ \\
\hline
\end{tabular}


enza mortality risk for the entire domain $R$ can be assessed. These maps make it possible to compare influenza risk variations during different seasons and at various geographical regions and identify these time periods/regions where the specified threshold is exceeded, and also quantify the level of such exceeding. Moreover, different thresholds may need to apply during different seasons to assure that adequate assessment is derived of local contributions to the statewide risk. E.g., if one season exhibits relatively lower risk variations than others, this season might be wrongly assumed not to have any contribution to the statewide influenza risk, assuming the same threshold is used as for the higher risk season.

\section{Results}

\subsection{Spatiotemporal mapping}

We assumed that the S/TRF $X(\boldsymbol{p})$ represents influenza mortality rates across space-time, where $\boldsymbol{p}=(\boldsymbol{s}, t) ; \boldsymbol{s}=\left(s_{1}, s_{2}\right)$ is the vector of longitudinal and latitudinal coordinates across California and $t$ is a temporal point to which we assign a $2 w i$ (recall that each seasonal period in 1997-2001 has 15 such points).

\subsubsection{Temporal profiles}

In Fig. 2 we plot the seasonal 1997-2001 variation of the mean value of the influenza mortality rates across
California (per $10^{5}$ individuals belonging to $a g \geq 65$ using $2 w i$ temporal units). The mean mortality values are considerably higher during the seasons 1997-1998 and 19992000 than those during the seasons 1998-1999 and 2000 2001. The former two seasons show similar temporal mortality profiles, and the same is true for the latter two seasons. In 1997-1998, the mean mortality rate increased considerably in late December $(t=8)$ and peaked in early January $(t=9)$, which is also valid for the season 19992000. On the other hand, both seasons 1998-1999 and 2000-2001 exhibited their mortality peaks from late January to early February $(t=11)$.

\subsubsection{Spatiotemporal covariance}

The covariance values of spatiotemporal influenza mortality rates were calculated simultaneously at the distances between every county pair and at the time intervals for every season separately. To the experimental covariance values, a theoretical space-time model of two exponential functions was fitted as follows

$c_{x}(r, \tau)=\sum_{i=1}^{2} c_{i} e^{-r / a_{s, i}-\tau / a_{t, i}}$,

where $r=\left|\boldsymbol{s}-\boldsymbol{s}^{\prime}\right|$ and $\tau=t-t^{\prime}$ are spatial and temporal distances between points $(\boldsymbol{s}, t)$ and $\left(\boldsymbol{s}^{\prime}, \boldsymbol{t}^{\prime}\right)$, the parameters $c_{i}$ (sills) offer a measure of the space-time variance of influenza mortality risk, and the $a_{s, i}$ and $a_{t, i}$ denote, respectively, the spatial and temporal correlations ranges of mortality rates. These model parameters were calculated for each
Fig. 2 Bi-weekly temporal profiles of the spatial mean influenza mortality rate per $10^{5}$ individuals of the $a g \geq 65$ group during the seasons 1997-2001
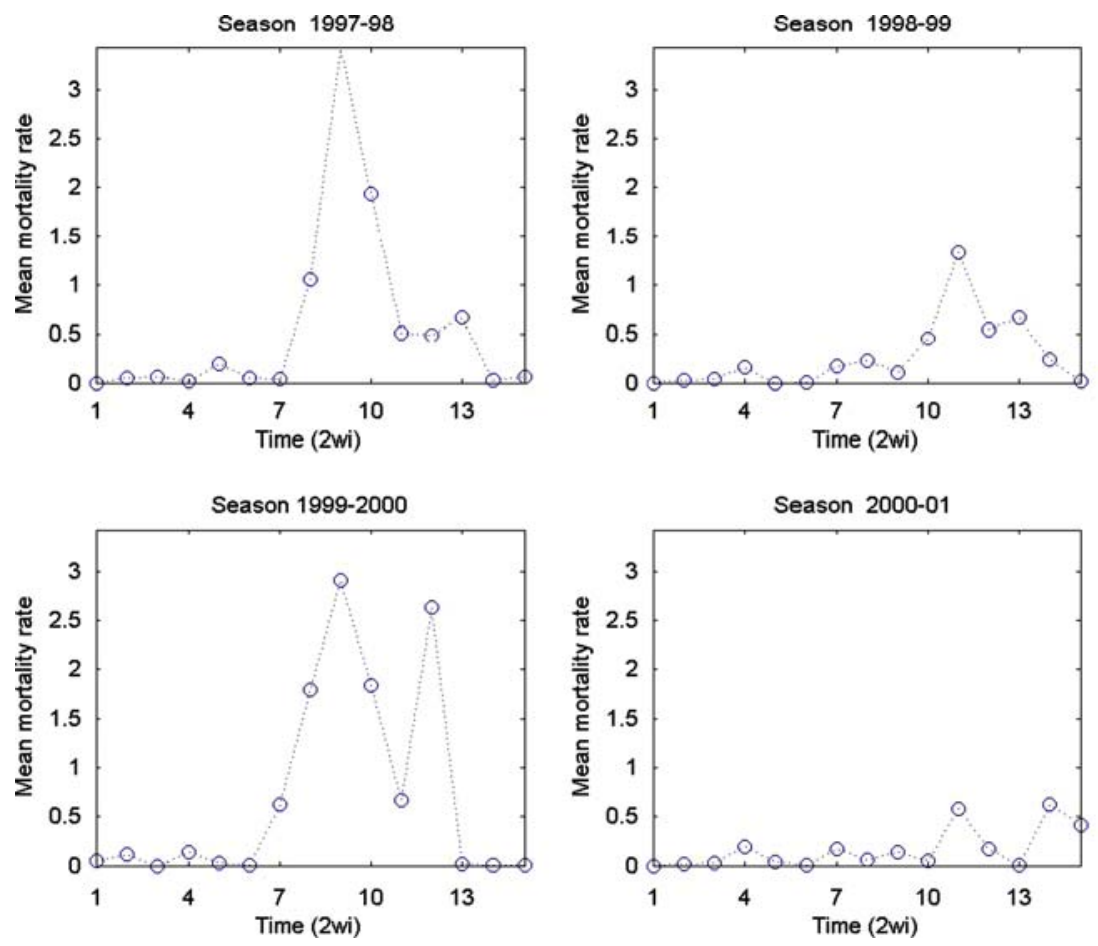
season, as shown in Table 3. The first exponential component $(i=1)$ in Eq. (3) mainly refers to local-range fitting, whereas the second component $(i=2)$ refers to long-range fitting. The model parameters in Eq. $\sim(3)$ were determined on the basis of the number of pairs $(r, \tau)$ across California and during the 15 time-intervals at which the experimental and theoretical covariance values showed a close match.

For illustration, in Fig. 3 we plot the influenza mortality covariance during the period 1999-2000 as a function of spatial lags $(K m)$ and time intervals $(2 w i)$. Note the linear shapes of the covariances at the space-time origin (implying considerable spatial and temporal variability of the mortality rates), and their asymptotic behaviors at large spatial lags and time intervals (implying a homogeneous and stationary behavior of the rates across space and time).

\subsubsection{Spatiotemporal soft data}

To account for the uncertainty of influenza mortality estimates, we transformed the information about death numbers into an interval soft data format. More specifically, the death rate is defined as the frequency with which people die at a geographical region (e.g., county) and at a specified time. The death rate has units of number of deaths per $10^{5}$ people per $2 w i$. Each US state reports mortality information from which it is possible to extract daily death counts at the county level. The measured death rates are actually the daily death counts $d_{i}$ due to influenza within each county $i$ divided by the number of residents $n_{i}$ in $10^{5}$ people units (based on US Census 1990 and 2000). If, e.g., there are $2 \times 10^{5}$ residents in county $i$, the number of residents (in $10^{5}$ people units) is $n_{i}=2$. It is often assumed that the ratio $d_{i} / n_{i}$ provides a reasonable approximation of the actual death rate for county $i$. However, $d_{i} / n_{i}$ is an uncertain quantity and, therefore, a more realistic representation of the situation was obtained by assuming that the actual death rate lies in the interval $\left(\frac{d_{i}-1}{n_{i}}, \frac{d_{i}+1}{n_{i}}\right)$; see Christakos and Serre (2000) for an explanation concerning this formulation. This type of uncertain measurements is referred to as soft interval data, and they are geographically associated with the centroid of each county.

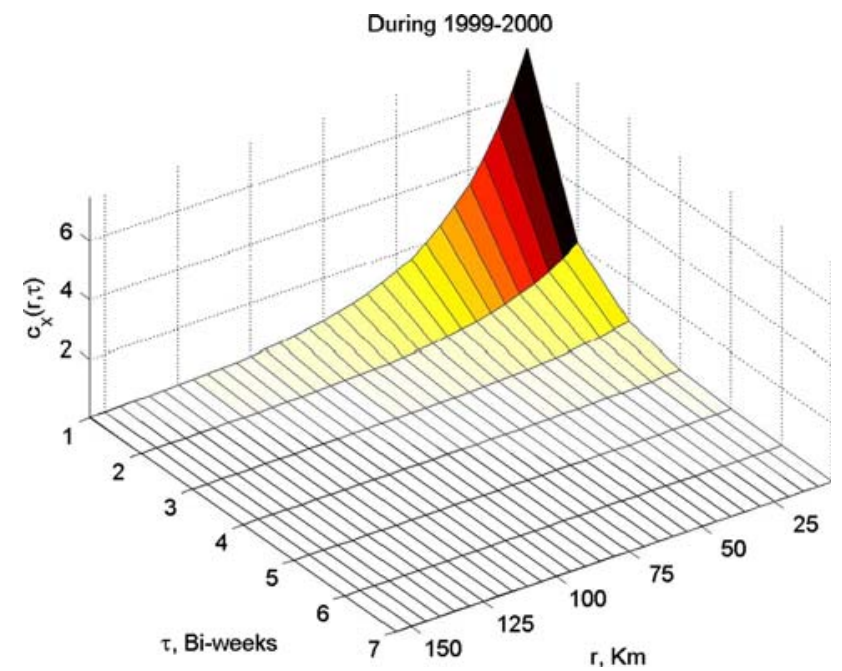

Fig. 3 Spatiotemporal covariance model of the influenza mortality rate per $10^{5}$ individuals of the $a g_{\geq 65}$ group in California during 1999-2000

\subsubsection{Estimates}

Using the general and specificatory knowledge bases above (theoretical covariance model, soft data, etc.), the BME method generated spatiotemporal maps of influenza mortality rates across California during the winter seasons of interest. The mean estimation errors were consistently less than $4 \times 10^{-6}$ units.

For visualization purposes, spatial maps of the influenza mortality rates are shown in Figs. 4, 5, 6, and 7. As before, the values are per $10^{5}$ individuals $(a g \geq 65)$ during the time periods 1997-2001 for (a) $t=7$, (b) $t=8$, (c) $t=9$ and (d) $t=10$ in $2 w i$ units (Table 1 provides the corresponding dates). These maps show that influenza mortality was, in general, high along the western coastline of California during most of the seasons considered, except during the 1998-1999 season. One may also notice that the influenza mortality maps for the seasons 19971998 and 1999-2000 are rather similar to each other (e.g., mortality spreads from the coastal areas to the northern parts of the state).

Table 3 The covariance model parameters

\begin{tabular}{|c|c|c|c|c|}
\hline Time period & 1997-1998 & 1998-1999 & 1999-2000 & 2000-2001 \\
\hline \multirow[t]{2}{*}{ Sill $\left(\times 10^{-11}\right)$} & $c_{1}=11.40$ & $c_{1}=1.26$ & $c_{1}=4.50$ & $c_{1}=0.54$ \\
\hline & $c_{2}=7.60$ & $c_{2}=0.84$ & $c_{2}=3.00$ & $c_{2}=0.09$ \\
\hline \multirow[t]{2}{*}{ Spatial range (in $\mathrm{Km}$ ) } & $a_{s, 1}=12$ & $a_{s, 1}=12$ & $a_{s, 1}=25$ & $a_{s, 1}=10$ \\
\hline & $a_{s, 2}=18$ & $a_{s, 2}=15$ & $a_{s, 2}=29$ & $a_{s, 2}=22$ \\
\hline \multirow[t]{2}{*}{ Temporal range (in $2 w i$ ) } & $a_{t, 1}=0.80$ & $a_{t, 1}=0.25$ & $a_{t, 1}=0.85$ & $a_{t, 1}=0.30$ \\
\hline & $a_{t, 2}=0.43$ & $a_{t, 2}=0.46$ & $a_{t, 2}=0.66$ & $a_{t, 2}=0.50$ \\
\hline
\end{tabular}


Fig. 4 Map of influenza mortality rate (per $10^{5}$

individuals of the $a g \geq 65$ group during 1997-1998) for a $t=7$ through $\mathbf{d} t=10$ (see Table $\sim 1$ for bi-weekly periods)
Fig. 5 Map of influenza mortality rate (per $10^{5}$ individuals of the $a g \geq 65$ group during 1998-1999) for a $t=7$ through $\mathbf{d} t=10$ (see Table 1 for bi-weekly periods) (a) Season 1997-1998 (t=7)
$44 \mathrm{~N}$

$42 \mathrm{~N}$

$40 \mathrm{~N}$

$38 \mathrm{~N}$

$36 \mathrm{~N}$

$34 \mathrm{~N}$

$32 \mathrm{~N}$

$-126 \mathrm{~W}$

$44 \mathrm{~N}$

$42 \mathrm{~N}$

$40 \mathrm{~N}$

$38 \mathrm{~N}$

$36 \mathrm{~N}$

$34 \mathrm{~N}$

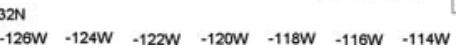

(a) Season 1998-1999 ( $\mathrm{t}=7)$

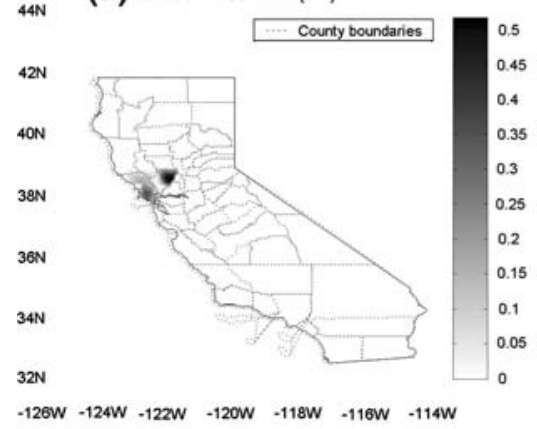

(c) Season 1998-1999 (t=9)

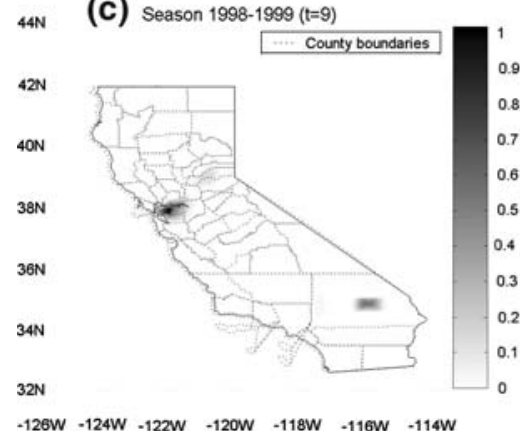

(b) Season 1997-1998 (t=8)

(d) Season 1997-1998 ( $t=10)$

$44 \mathrm{~N}$

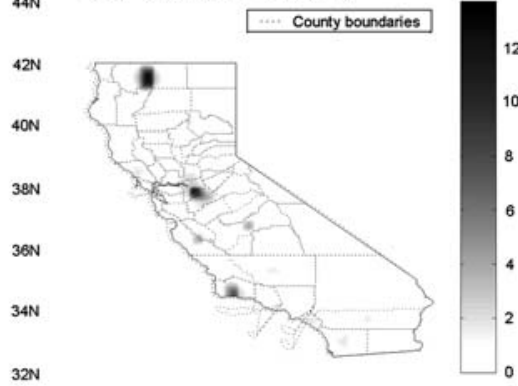

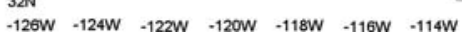

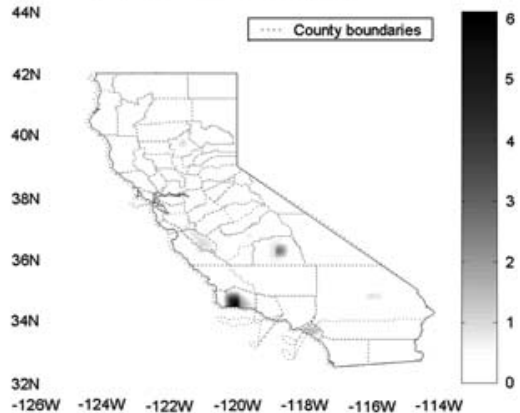

$44 \mathrm{~N}$

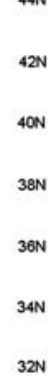

$32 \mathrm{~N}$

$-126 W-124 W-122 W-120 W-118 W-116 W-114 W$ (b) Season 1998-1999 ( $t=8)$

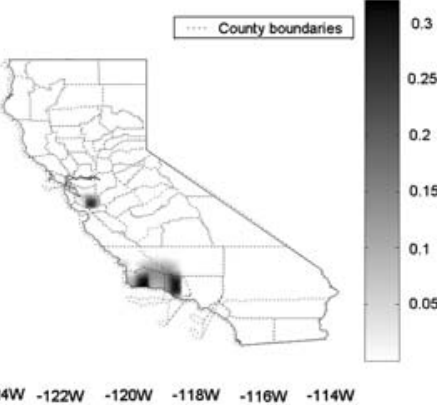

(d) Season 1998-1999 (t=10)

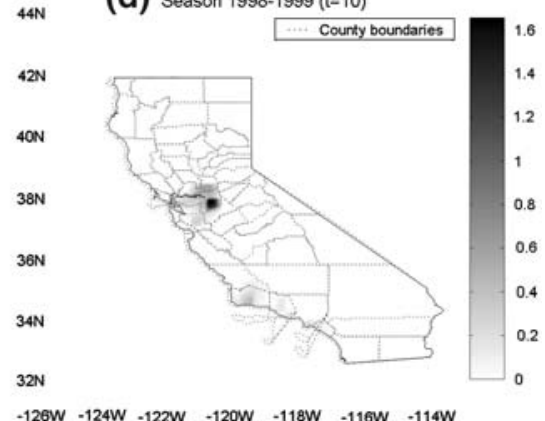

For numerical illustration purposes, the temporal mortality plots shown in Fig. 8 refer to influenza mortality rates estimated at the Los Angeles centroid (per $10^{5}$ individuals belonging to the $a g \geq 65$ group) during different seasons. Notice that the magnitude of the mortality rate varies considerably.
3.2 Spatiotemporal risk patterns

\subsubsection{Statewide risk patterns}

Based on the spatiotemporal BME analysis, influenza mortality risks were calculated across space and time from 
Fig. 6 Map of influenza mortality rate (per $10^{5}$

individuals of the $a g \geq 65$ group during 1999-2000) for a $t=7$ through $\mathbf{d} t=10$ (see Table 1 for bi-weekly periods)
Fig. 7 Map of influenza mortality rate (per $10^{5}$ individuals of the $a g \geq 65$ group during 2000-2001) for a $t=7$ through $\mathbf{d} t=10$ (see Table 1 for bi-weekly periods) (a) Season 1999-2000 (t=7)

$44 \mathrm{~N}$

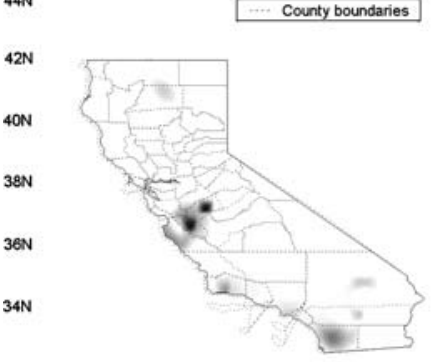

$32 \mathrm{~N}$

$-126 W-124 W-122 W-120 W-118 W-116 W-114 W$

(C) Season 1999-2000 ( $\mathrm{t}=9)$

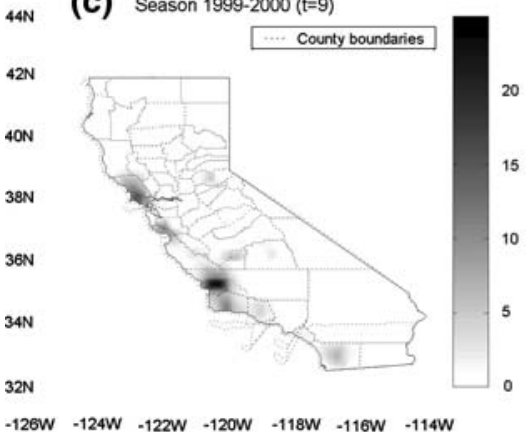

$-128 W-124 W-122 W-120 W-118 W-116 W-114 W$
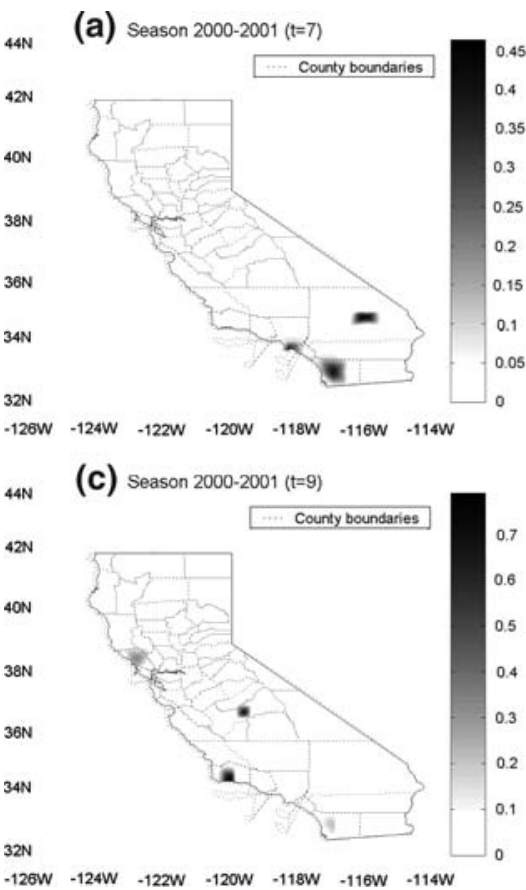

(b) Season 1999-2000 (t=8) $44 \mathrm{~N}$

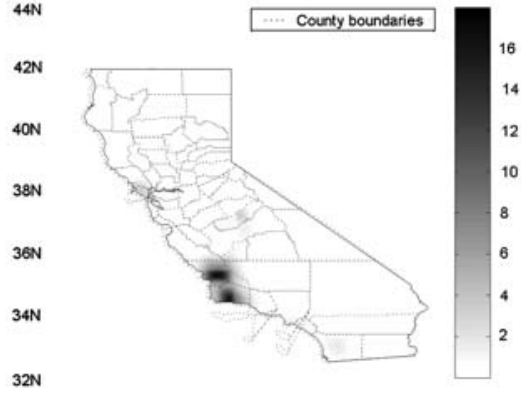

$-126 \mathrm{~W}-124 \mathrm{~W}-122 \mathrm{~W}-120 \mathrm{~W}-118 \mathrm{~W}-116 \mathrm{~W} \quad-114 \mathrm{~W}$

(d) Season 1999-2000 ( $t=10)$

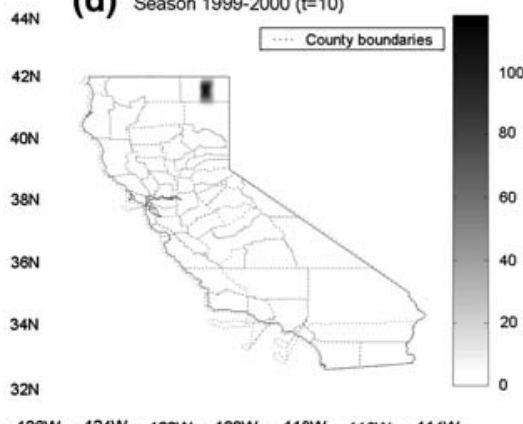

$-126 W-124 W-122 W-120 W-118 W-116 W-114 W$

(b) Season 2000-2001 (t=8)

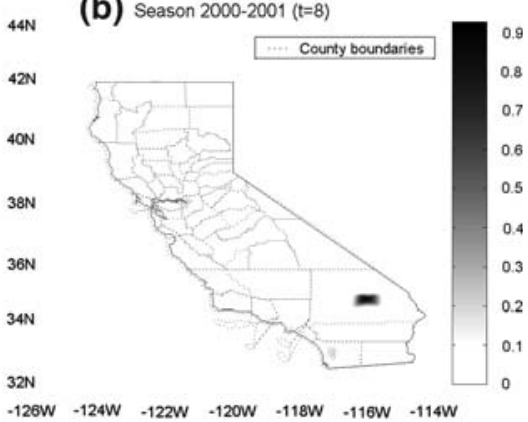

(d) Season 2000-2001 (t=10)

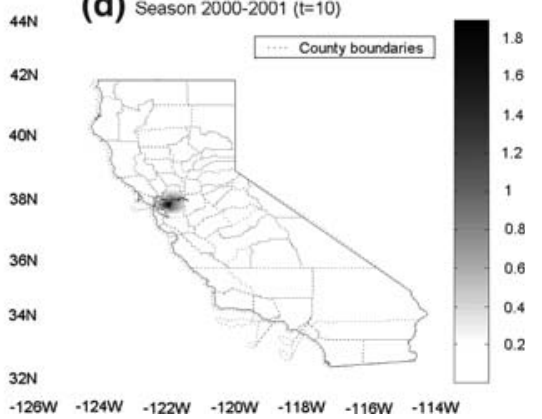

the mortality rate estimation results. It was found that the 1997-1998 and 1999-2000 seasons had similar temporal/ geographical patterns of influenza mortality risk, duration periods, etc. Note that the mortality pattern during the seasons 1998-1999 and 2000-2001 was characterized by a 1-month delay compared to the other two seasons. Also, compared to other seasons, the 2000-2001 season showed little temporal variation in influenza mortality risks. These results are represented in the following plots.

In Fig. 9 we plot the seasonal state risk patterns in terms of area percentages associated with influenza mortality estimates exceeding the seasonal thresholds. We considered two thresholds (in deaths per $10^{7}$ individuals $a g \geq 65$ ): 55 for seasons 1997-1998 and 1999-2000, and 20 for 
Fig. 8 The influenza mortality rate estimates and soft data at the centroid of Los Angeles (per $10^{5}$ individuals of the $a g \geq 65$ group)
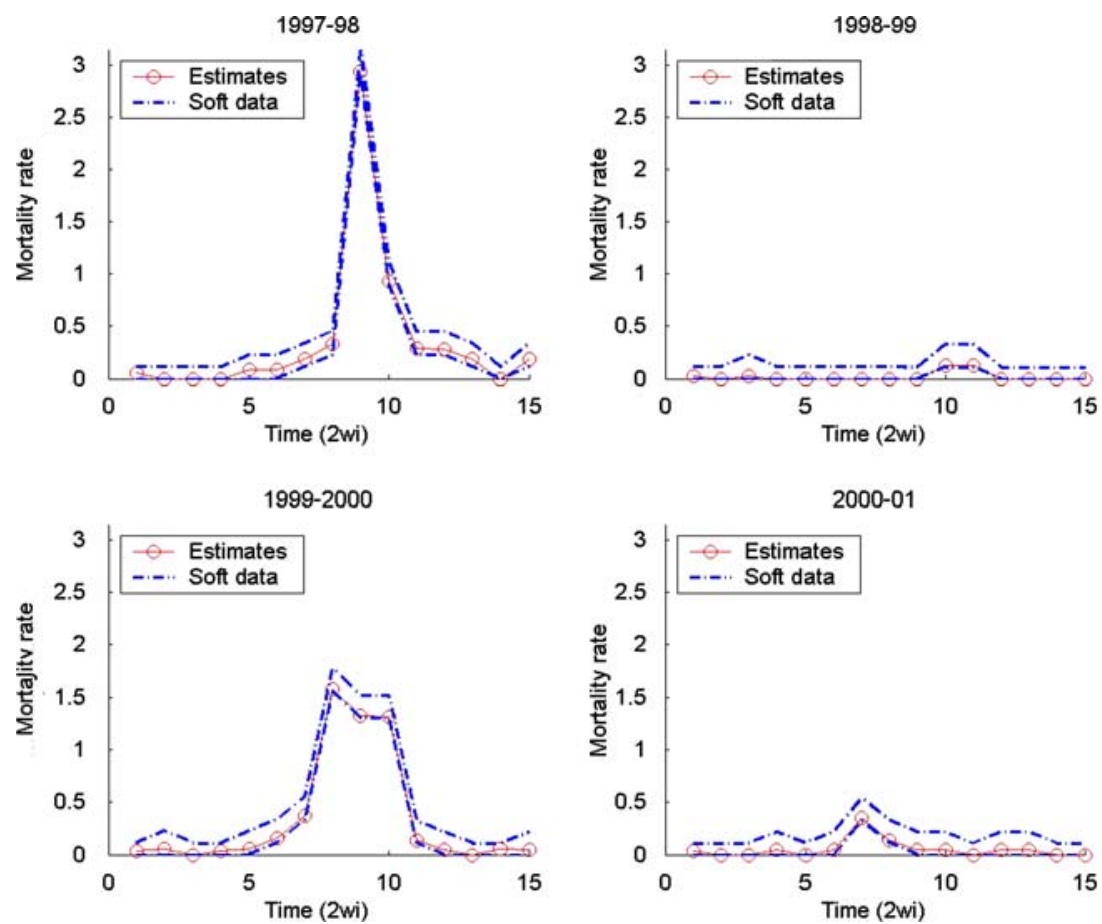

seasons 1998-1999 and 2000-2001. These seasonal thresholds were selected so that, not only influenza mortality rates are significantly high at the chosen statistical level $(\alpha=0.05)$ but also that different seasons can be compared to each other with respect to variation in the risk level and its duration period. Among the years 1997-2001, the statewide influenza mortality risk was highest during the season 1999-2000, especially the months December to January ( $t=7-10$ in $2 w i$ units). Then, the seasonal influenza mortality risks decreased and after $t=10$ the risks were statistically significant at $\alpha=0.05$ (seasonal threshold

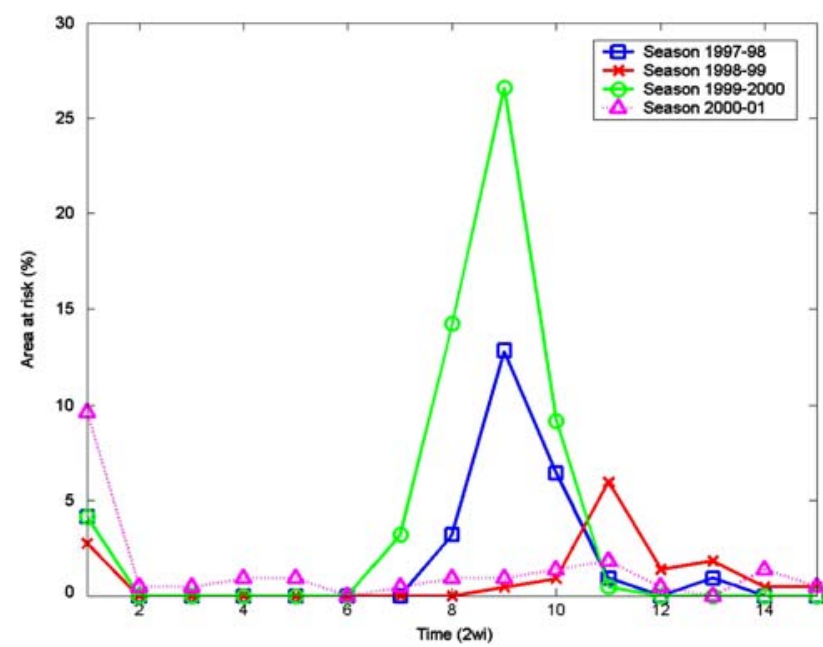

Fig. 9 Statewide risk (i.e., statewide area at risk; in \%) during the seasons 1997-2001 is 55) in less than $5 \%$ of the state. Similar patterns were observed during the season 1997-1998. At the statistical significance level considered, about $27 \%$ of the state exhibited an influenza mortality risk above the seasonal threshold, especially in early January $2000(t=9)$, and $13 \%$ of the state during the season 1997-1998. Only $6 \%$ of the state experienced influenza mortality risk above the seasonal threshold for a short time period during 19981999 (note that a lower threshold was considered in this season than in the 1997-1998 and 1999-2000 seasons at the significance level $\alpha=0.05$ ). In the season 1998-1999 noticeable statewide risk was observed mostly in early February $(t=11)$ and it decreased shortly after that. During the season 2000-2001, less than 5\% of the state was found to be above the seasonal threshold even during the months of December through February.

Table 3 presents the covariance parameter values during the four study periods. Some useful information can be extracted from the fitted covariance models. The variation of mortality rates is higher during the periods 1997-1998 and 1999-2000, which can be associated with the larger covariance sills during these periods. The difference in the spatial ranges between the periods 1997-1998 and 19992000 is linked to the fact that a wider geographical spreading of influenza occurred during the period 19992000 than during 1997-1998. In other words, the influenza distribution is more centralized in 1997-1998 than in 1999-2000. The temporal ranges (scales) during these four years are relatively uniform (less than 2 weeks in magnitude), which may be linked to influenza transmission 
dynamics with less than 2 weeks latent period (Chowell et al. 2006).

\subsubsection{Regional risk contributions}

To study any repeated patterns in the spread of influenza risk across the state we used the risk measure of Eq. (2). We calculated first regional risk contributions to statewide risk along the regions shown in Fig. 1 and then compared them for different seasons. Figure 10 shows the variation of regional influenza risk versus time during different seasons. During the 1997-1998 season (Figs. 4, 9, 10a), $41 \%$ of the statewide risk occurred in the west-central California in late December $(t=8)$. The remaining $59 \%$ of the risk for that period was observed in the south and eastcentral regions of the state. As risk contribution from the central region decreased, the southern region generated as much as $48 \%$ of the statewide risk in early January 1998 $(t=9)$. When the statewide risk started to decrease in late January 1998 ( $t=10$ in Fig. 9), the west-central and northern parts of the state rather increased their contributions to the statewide risk. After January 1998, influenza risk was occurring mainly in the west-central part of the state.

The initial increase of the statewide risk in late January 1999 ( $t=10$ in Figs. 5d, 9 and 10b) was contributed mainly by the west-central region of the state. There was a large increase in statewide risk in early February $(t=11$ in Fig. 10b), of which $61 \%$ was contributed mainly by the east-central part of the state, whereas the remaining risk was distributed in the west-central and southern parts of the state. As the statewide risk declined in late February 1999 $(t=12)$, the influenza risk spread into the northern and southern parts of the state, but not into the central part of the state, until early March. It is noteworthy that the statewide risk was low enough to be ignored after early February 1999 ( $t=11$ in Fig. 9).

During season 1999-2000 (Figs. 6, 9, 10c), 85\% of the statewide risk in the beginning of December $1999(t=7)$ occurred in the west-central part of the state, and $15 \%$ in the southern part of the state. Rapid increase in the spread of statewide risk occurred in the southern part of the state: 64 and 49\%, respectively, in late December $1999(t=8)$ and in early January $2000(t=9)$. The central part of the state contributed the remaining percentage of the statewide risk in late December through early January ( $t=8$ and 9). The northern part of the state contributed $35 \%$ of the statewide risk in late January $2000(t=10)$, and the westcentral and southern parts contributed the remaining percentage of the risk for that period. The statewide risk after January 2000 occurred mainly in the western part of the state (this was also the case for the other seasons).

The season 2000-2001 (Figs. 7, 9, 10d) has the interesting feature. In most time intervals considered the statewide risk occurred in one regional part of the state rather than in multiple regional parts. $100 \%$ of the statewide risk occurred only in the southern part of California at $t=2$ and 7, west-central California at $t=3$ and 12, and northern California at $t=4,11$ and 15 (Fig. 10d). In addition, no significant risk occurred in the east-central
Fig. 10 Regional risk contributions (in \%) to the statewide risk during each season: a 1997-1998, b 19981999, c 1999-2000 and d 20002001
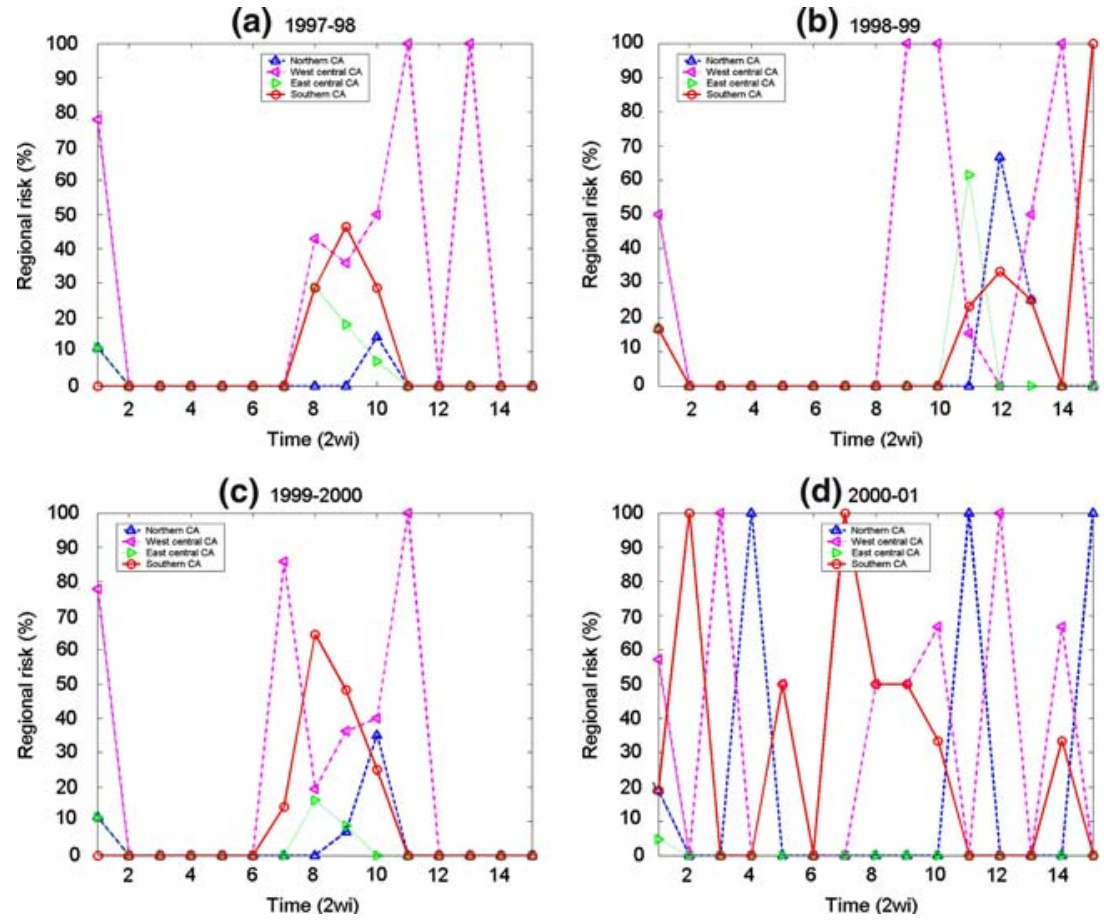
California. As we see in Fig. 9, during the season 20002001 the statewide risk was found to be relatively small compared to other seasons.

\section{Discussion}

We studied influenza mortality risk in the state of California during the winter seasons of the years 1997-2001. Mortality rates and the associated risks were represented in terms of spatiotemporal random fields and predictions were generated using BME techniques. Influenza risk was assessed in terms of the size of the local region in which influenza mortality exceeded a certain threshold (number of deaths), and its spread across the state was considered at 2-week intervals. Our space-time analysis detected some influenza risk patterns that were shared by different winter seasons. During each season, the spread of influenza risk in California started mainly in the west-central part of the state. In the context of health planning, this event could be considered as a warning sign that an influenza statewide epidemic may follow in a couple of weeks. After its initial spread phase, the influenza risk in the west-central part of the state typically started to decrease and at the same time the risk at the southern part started to increase noticeably. During the high risk seasons (1997-1998 and 1999-2000) significant risk values were initially observed at the month of December. Higher influenza risk distributions were found to last longer than low risk distributions. Further research is needed to identify other important factors associated with the influenza risk dynamics across the state of California, so that influenza epidemics can be efficiently prevented.

Acknowledgments We would like to thank Dr. RA Olea of USGS for his valuable suggestions. This research was supported by grants from the Oak Ridge National Lab (OR7865-001.01), the Fred J. Hansen Institute, SDSU Foundation, California (54266A P3590), the Office of the Vice President for Research at the University of Michigan and the National Oceanographic and Atmospheric Administration (NA16GP23361).

\section{References}

Anderson RM, May RM (1991) Infectious disease of humans: dynamics and control. Oxford University Press, Oxford

Besag J, Newell J (1991) The detection of clusters in rare diseases. J R Stat Soc Ser A 154:143-155

Bogaert P (1996) Comparison of kriging techniques in a space-time context. Math Geol 28(1):73-86

Choi KM, Christakos G, Wilson ML (2006) El Niño effects on influenza mortality risks in the State of California. J Public Health 120:505-516

Chowell G, Ammonb CE, Hengartnera NW, Hymana JM (2006) Transmission dynamics of the great influenza pandemic of 1918 in Geneva, Switzerland: assessing the effects of hypothetical interventions. J Theoret Biol 241(2):193-204

Christakos G (1990) Random field modelling and its applications in stochastic data processing. Applied Sciences, PhD Thesis Harvard University, Cambridge, MA

Christakos G (1991) On certain classes of spatiotemporal random fields with application to space-time data processing. IEEE Syst Man Cybern 21(4):861-875

Christakos G (1992) Random field models in earth sciences. Academic, San Diego, CA (Out of Print.) New edition, Dover, Mineola, NY, 2005

Christakos G, Bogaert P (1996) Spatiotemporal analysis of springwater ion processes derived from measurements at the Dyle Basin in Belgium. IEEE Trans Geosci Remote Sens 34(3):626642

Christakos G (1998) Spatiotemporal information systems in soil and environmental sciences. Geoderma 85(2-3):141-179

Christakos G (2000) Modern spatiotemporal geostatistics. Oxford University Press, New York

Christakos G, Serre ML (2000) A spatiotemporal study of exposurehealth effect associations. J Expos Anal Environ Epidemiol 10(2):168-187

Christakos G, Bogaert P, Serre ML (2002) Temporal GIS with CDROM. Springer, New York

Christakos G, Hristopulos DT (1998) Spatiotemporal environmental health modelling: a tractatus stochasticus. Kluwer, Boston

Christakos G, Olea RA, ML Serre, HL Yu, Wang L (2005) Interdisciplinary public health reasoning and epidemic modelling: the case of black death. Springer, New York

Cliff AD (1995) Incorporating spatial components into models of epidemic models. In: Mollison D, Moffatt HK (eds) Epidemic models: their structure and relation to data. Cambridge Univ Press, Cambridge, pp 119-149

Cliff AD, Haggett P (1988) Atlas of disease distributions: analytic approaches to epidemiological data. Blackwell, Oxford

Cliff AD, Haggett P, Ord JK (1986) Spatial aspects of influenza epidemics. Pion, London

Cressie N, Huang HC (1999) Classes of nonseparable, spatiotemporal stationary covariance functions. J Am Stat Assoc 94:1330-1340

Cuzick J, Edwards R (1990) Spatial clustering for inhomogeneous populations. J R Stat Soc Ser B 52:73-104

Death Statistical Master Files (DSMF) (1997-2001) Center for Health Statistics, Sacramento, CA

Earn, DJD, Dushoff J, Levin SA (2002) Ecology and evolution of the flu. TRENDS Ecol Evol 17(7):334-340

Ferguson MP, Neil A, Galvani R, Bush M (2003) Ecological and immunological determinants of influenza evolution. Nature 422:428-433

Golledge RG (2002) The nature of geographical knowledge. Ann Assoc Am Geogr 92(1):1-14

Goodall C, Mardia KV (1994) Challenges in multivariate spatiotemporal modeling. In: Proceedings of the XVIIth international biometric confererence, Hamilton, 8-12 August 1994, pp 1-17

Haas TC (1995) Local prediction of spatio-temporal process with an application to wet sulfate deposition. J Am Stat Assoc 90:11891199

Haggett P (2000) The geographical structure of epidemics. Clarendon Press, Oxford

Harper SA, Fukuda K, Uyeki TM, Cox NJ, Bridges CB (2004) Prevention and control of influenza recommendations of the advisory committee on immunization practices. Morbidity and Mortality Weekly Report 53:1-40

Kyriakidis PC, Journel AG (1999) Geostatistical space-time models: a review. Math Geol 31(6):651-684 
Lui KJ, Kendal AP (1987)119-149 Impact of influenza epidemics on mortality in the United States. Am J Public Health 77:712-716

May RM, Anderson RM (1984) Spatial heterogeneity and the design of immunization programs. Math Biosci 72:83-111

Openshaw S, Charlton M (1987) A mark 1 Geographical analysis machine for the automated analysis of point data sets. Int $\mathbf{J}$ Geogr Inform Syst 1:335-358
Openshaw S, Craft AW, Charlton M, Birch JM (1988) Investigation of leukaemia clusters by use of geographical analysis machine. The Lancet 272-273

Viboud C, Boelle P-Y, Carrat F, Valleron A-J, Flahault A (2003) Prediction of the spread of influenza epidemics by the method of analogues. Am J Epidemiol 158: 996-1006 